\title{
The First Total Synthesis of Sporiolide A
}

\author{
Yuguo Du, ${ }^{*} \dagger$ Qi Chen, ${ }^{\dagger}$ and Robert J. Linhardt ${ }^{\ddagger}$ \\ State Key Laboratory of Environmental Chemistry and Ecotoxicology, Research Center for \\ Eco-Environmental Sciences, Chinese Academy of Sciences, Beijing 100085, China, and Departments of \\ Chemistry, Biology, and Chemical and Biological Engineering, Rensselaer Polytechnic Institute, Troy,
} New York 12180

duyuguo@rcees.ac.cn

Received July 26, 2006

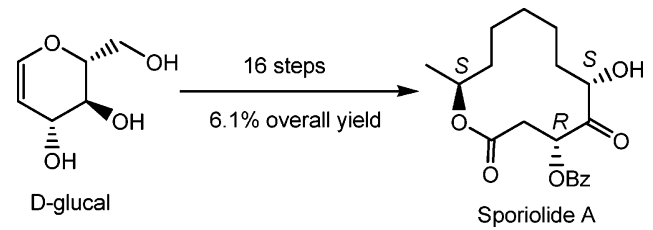

The first total synthesis of the natural cytotoxic agent sporiolide A has been accomplished from D-glucal in 16 steps with $6.1 \%$ overall yield. Carbohydrates were applied as the chiral templates to manipulate the absolute configuration during the synthesis. Pyridinium chlorochromate (PCC)-promoted transformation of the cyclic enol-ether to lactone, followed by Yamaguchi esterification and intramolecular ring closure metathesis, greatly facilitates synthesis of the target compound.

\section{Introduction}

Marine fungi are attracting increasing attention as a potential source of new pharmaceuticals and pharmaceutical leads. ${ }^{1}$ These leading compounds include fungal metabolites containing 12membered lactone rings such as sporiolides [A (1), B (2)] and pandangolide 1 (3) isolated from the cultured broth of $\mathrm{Cla}$ dosporium sp. from an Okinawan marine brown alga Actinotrichia fragilis and the Red Sea sponge Niphates rowi (Figure 1). ${ }^{2}$ Sporiolides A and B exhibit cytotoxicity against L1210 cells with $\mathrm{IC}_{50}$ values of 0.13 and $0.81 \mu \mathrm{g} / \mathrm{mL}$, respectively. Importantly, sporiolide A shows antifungal activity against Candida albicans (MIC $16.7 \mu \mathrm{g} / \mathrm{mL}$ ), Cryptococcus neoformans $(8.4 \mu \mathrm{g} / \mathrm{mL})$, Aspergillus niger $(16.7 \mu \mathrm{g} / \mathrm{mL})$, and Neurospora crassa $(8.4 \mu \mathrm{g} / \mathrm{mL})$, together with antibacterial activity against Micrococcus luteus (16.7 $\mu \mathrm{g} / \mathrm{mL})$. In 2004, Kobayashi and coworkers proposed a chemical structure of sporiolide A corresponding to 3-O-benzoyl pandangolide 1 based on spectroscopic data, ${ }^{3}$ although the absolute configuration of pandangolide 1

\footnotetext{
$\dagger$ Chinese Academy of Sciences.
}

$¥$ Rensselaer Polytechnic Institute.

(1) (a) Blunt, J. W.; Copp, B. R.; Munro, M. H. G.; Northcote, P. T.; Prinsep, M. R. Nat. Prod. Rep. 2004, 21, 1-49. (b) Faulkner, D. Nat. Prod. Rep. 2002, 19, 1-48. (c) Blunt, J. W.; Copp, B. R.; Munro, M. H. G.; Northcote, P. T.; Prinsep, M. R. Nat. Prod. Rep. 2005, 22, 15-61. (d) Blunt, J. W.; Copp, B. R.; Munro, M. H. G.; Northcote, P. T.; Prinsep, P. R. Nat. Prod. Rep. 2006, 23, 26-78. (e) Tsuda, M.; Mugishima, T.; Komatsu, K.; Sone, T.; Tanaka, M. J. Nat. Prod. 2003, 66, 412-415.

(2) Kobayashi, J.; Tsuda, M. Phytochem. Rev. 2004, 3, 267-274.

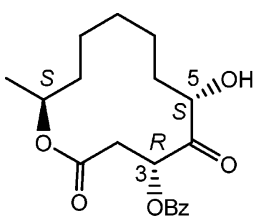

1 (Sporiolide A)

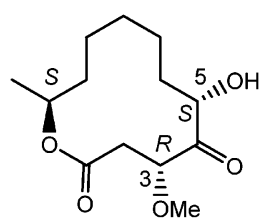

2 (Sporiolide B)

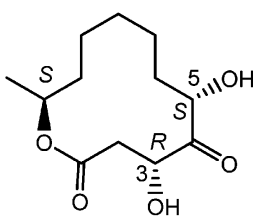

3 (Pandanglide 1)
FIGURE 1. Structures of macrolides from marine-derived fungus Cladosporium species.

was not determined until 2005. ${ }^{4}$ The potential biological importance, as well as the uncertainty of its absolute structural configuration, marked sporiolide A as a synthetic target for us. Herein, we report our efforts leading to the first total synthesis of sporiolide A (1) using chiral carbohydrate templates.

\section{Results and Discussion}

As outlined in Scheme 1, we decided to prepare sporiolide A (1) from olefinic intermediate 4 that we envisaged would be made available from intramolecular ring closure metathesis (RCM) of diene $\mathbf{5}$ after esterification of acid $\mathbf{6}$ and alcohol $\mathbf{7 .}$ Key intermediate $\mathbf{6 a}$ could be derived from cyanated D-xylose derivative $\mathbf{8}$ to afford the proposed absolute configurations at

(3) Shigemori, H.; Kasai, Y.; Komatsu, K.; Tsuda, M.; Mikami, Y.; Kobayashi, J. Mar. Drugs 2004, 2, 164-169.

(4) Gesner, S.; Cohen, N.; Iian, M.; Yarden, O.; Carmeli, S. J. Nat. Prod. 2005, 68, 1350-1353. 
SCHEME 1. Retrosynthetic Analysis of Sporiolide A (1)
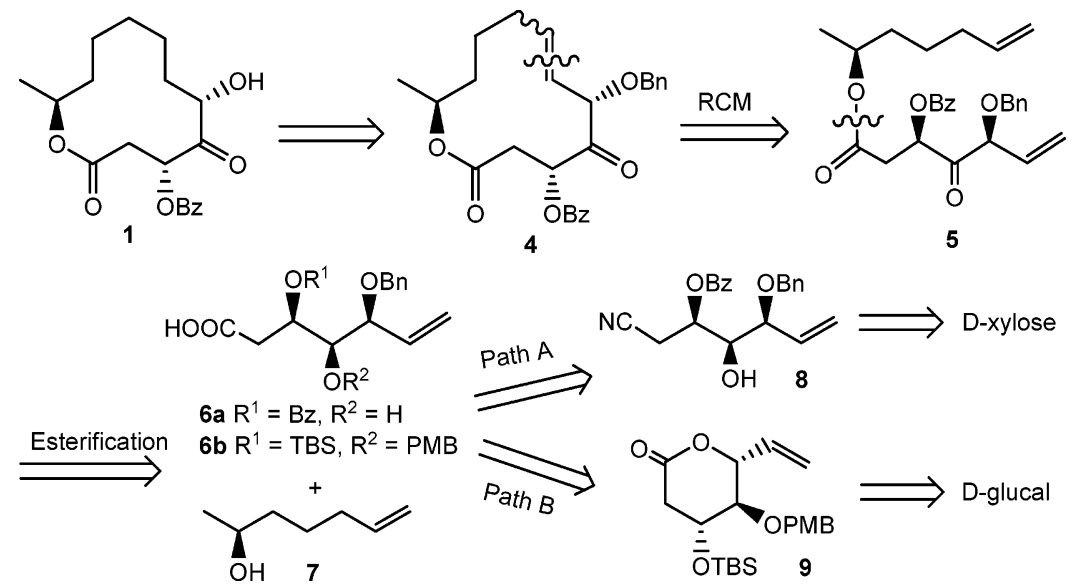

SCHEME 2. Attempted Synthesis of Sporiolide A from d-Xylose ${ }^{a}$

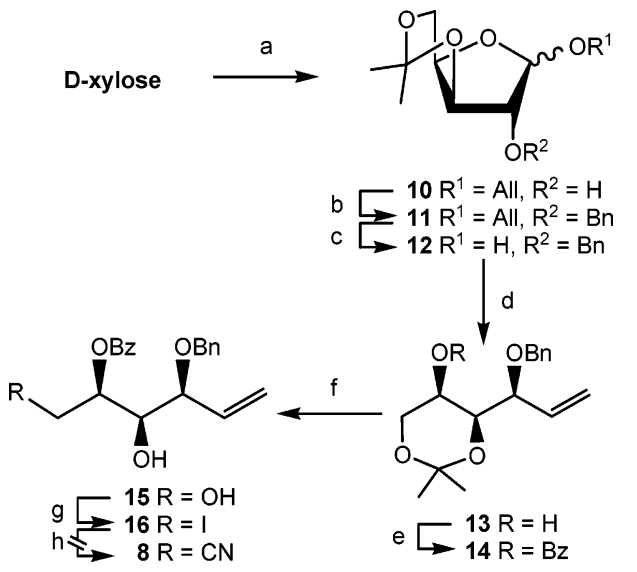

\begin{abstract}
${ }^{a}$ Reagents and conditions: (a) ref 5; (b) BnBr, NaH, DMF, 2 h, 95\% $(\alpha / \beta=1.5 / 1)$; (c) (i) t-BuOK, DMSO, $80{ }^{\circ} \mathrm{C}, 15 \mathrm{~min}$; (ii) $\mathrm{Hg}(\mathrm{OAc})_{2}, \mathrm{THF} /$ $\mathrm{H}_{2} \mathrm{O}, 30 \mathrm{~min}, 84 \%$ for two steps $(\alpha / \beta=2 / 1)$; (d) $\mathrm{BuLi}, \mathrm{Ph}_{3} \mathrm{PCH}_{3} \mathrm{Br}$, THF, $-50{ }^{\circ} \mathrm{C}$ to rt, $12 \mathrm{~h}, 75 \%$; (e) BzCl, Pyr, DMAP, $2 \mathrm{~h}, 98 \%$; (f) $\mathrm{TsOH} \cdot \mathrm{H}_{2} \mathrm{O}$, $\mathrm{MeOH} / \mathrm{H}_{2} \mathrm{O}, 3$ h, 95\%; (g) $\mathrm{Ph}_{3} \mathrm{P}, \mathrm{I}_{2}$, imidazole, THF, 12 h, 81\%; (h) KCN,
\end{abstract} DMF, rt to $150{ }^{\circ} \mathrm{C}$.

C-3 and C-5 in sporiolide A (path A, Scheme 1). Alternatively, as our subsequent investigation would make clear, acid $\mathbf{6 b}$ could be obtained from the appropriately functionalized lactone $\mathbf{9}$, which could be prepared from commercially available D-glucal (path B, Scheme 1).

Initially, we tried to prepare key intermediate $\mathbf{8}$ starting from D-xylose as illustrated in Scheme 2. Thus, benzylation of known D-xylofuranosyl derivative $\mathbf{1 0}^{5}$ and subsequent deallylation $(t$ $\mathrm{BuOK}$ in DMSO and $\mathrm{Hg}(\mathrm{OAc})_{2}$ in $\left.\mathrm{THF} / \mathrm{H}_{2} \mathrm{O}\right)^{6}$ gave hemiacetal 12 in good yield (80\% from 10). Standard Wittig reaction of 12 with methyltriphenylphosphonium bromide in anhydrous THF at $-50{ }^{\circ} \mathrm{C}$ generated olefin $\mathbf{1 3}$, in which the hydroxyl group was benzoylated using $\mathrm{BzCl}$ and pyridine $(\rightarrow \mathbf{1 4})$. Acid hydrolysis of 14 with $\mathrm{TsOH}$ hydrate $(\rightarrow \mathbf{1 5})$ and regioselective iodination ${ }^{7}$ with $\mathrm{Ph}_{3} \mathrm{P}$ and $\mathrm{I}_{2}$ furnished iodide $\mathbf{1 6}$ in an isolated yield of $57 \%$ over 4 steps. Attempted $\mathrm{S}_{\mathrm{N}} 2$ substitution of iodide to the cyano group with $\mathrm{KCN}$ using many known procedures was

(5) Ireland, R. E.; Norbeck, D. W. J. Am. Chem. Soc. 1985, 107, 32793285 .

(6) Gigg, R.; Warren, C. D. J. Chem. Soc. C 1968, 1903-1911.

(7) Uehara, H.; Oishi, T.; Inoue, M.; Shoji, M.; Nagumo, Y.; Kosaka, M.; Brazidec, J. L.; Hirama, M. Tetrahedron 2002, 58, 6493-6512.

fruitless. ${ }^{8}$ However, in a model study, this transformation was successfully carried out when methyl ether was used in place of the benzoate group. Furthermore, if the benzoate group was replaced by 4-methoxybenzyl ether, the desired compound failed to form under the same reaction conditions. Since the literature ${ }^{9}$ suggested that this problem might be hard to overcome, we focused our attention on path B of Scheme 1, as shown in Scheme 3.

D-Glucal was regioselectively silylated at the 3- and 6-positions according to a literature method ${ }^{10}$ to give compound $\mathbf{1 7}$, which was then treated with $\mathrm{PMBCl}$ and $\mathrm{NaH}$ in $\mathrm{DMF}$, affording fully protected glucal 18 (75\% yield from D-glucal). Regioselective desilylation of $\mathbf{1 8}$ with $\mathrm{HF}$ - pyridine complex ${ }^{11}$ gave $\mathbf{1 9}$, providing a free primary hydroxyl, which was then oxidized by Swern oxidation and alkenated under Witttig reaction conditions $(\rightarrow \mathbf{2 0})$. This D-glucal derivative $\mathbf{2 0}$ was subjected to pyridinium chlorochromate (PCC)-promoted transformation of cyclic enol ether to lactone ${ }^{12}$ at $45^{\circ} \mathrm{C}$ in the presence of silica gel to give key intermediate $\mathbf{9}$ in $74 \%$ yield. Treatment of $\mathbf{9}$ with $\mathrm{NaOMe}$ in $\mathrm{MeOH}$ was formed methyl ester 21, in which free $\mathrm{OH}$ was blocked by a benzyl group using benzyltrichloroacetimdate $^{13}$ and trimethylsilyltrifluoromethanesulfonate (TMSOTf), affording compound 22. Hydrolysis of $\mathbf{2 2}$ with $\mathrm{LiOH}$, followed by esterification with $(S)$-6-hepten-2-ol (7) ${ }^{14}$ under Yamaguchi's conditions, ${ }^{15}$ afforded diene derivative 23, which was subjected to desilylation with tetrabutylammonium fluoride (TBAF) $(\mathbf{\rightarrow 2 4})$, benzoylation with $\mathrm{BzCl}$ in pyridine $(\rightarrow \mathbf{2 5})$, cleavage of PMB group with $\mathrm{DDQ}^{16}(\rightarrow \mathbf{2 6})$, and DessMartin periodinane oxidation, ${ }^{17}$ to form ketone diene intermedi-

(8) Muratake, H.; Natsume, M. Tetrahedron 2006, 62, 7056-7070. 9788.

(9) Kabeya, M.; Hamada, Y.; Shiori, T. Tetrahedron 1997, 53, 9777-

(10) Kinzy, W.; Schmidt, R. R. Tetrahedron Lett. 1987, 28, 1981-1984. (11) Crouch, R. D. Tetrahedron 2004, 60, 5833-5871.

(12) (a) Rollin, P.; Sinay,, P. Carbohydr. Res. 1981, 98, 139-142. (b) Roth, B. D.; Roark, W. H. Tetrahedron Lett. 1988, 29, 1255-1258. (c) Baba, T.; Huang, G.; Isobe, M. Tetrahedron 2003, 59, 6851-6872. (c) Piancatelli, G.; Scettri, A.; D'Auria, M. Tetrahedron Lett. 1977, 18, 34833484.

(13) Du, Y.; Liu, J.; Linhardt, R. J. J. Org. Chem. 2006, 71, 12511253.

(14) Takahata, H.; Yotsui, Y.; Momose, T. Tetrahedron 1998, 54, 13505-13516.

(15) Inanaga, J.; Hirata, K.; Saeki, H.; Katsuki, T.; Yamaguchi, M. Bull. Chem. Soc. Jpn. 1979, 52, 1989-1993.

(16) (a) Oikawa, Y.; Tanaka, T.; Horita, K.; Yonemitsu, O. Tetrahedron Lett. 1984, 25, 5397-5400. (b) Tanaka, T.; Oikawa, Y.; Hamada, T.; Yonemitsu, O. Tetrahedron Lett. 1986, 27, 3651-3654.

(17) Dess, P. B.; Martin, J. C. J. Org. Chem. 1983, 48, 4155-4156. 


\section{SCHEME 3. Total Synthesis of Sporiolide A (1) ${ }^{a}$}
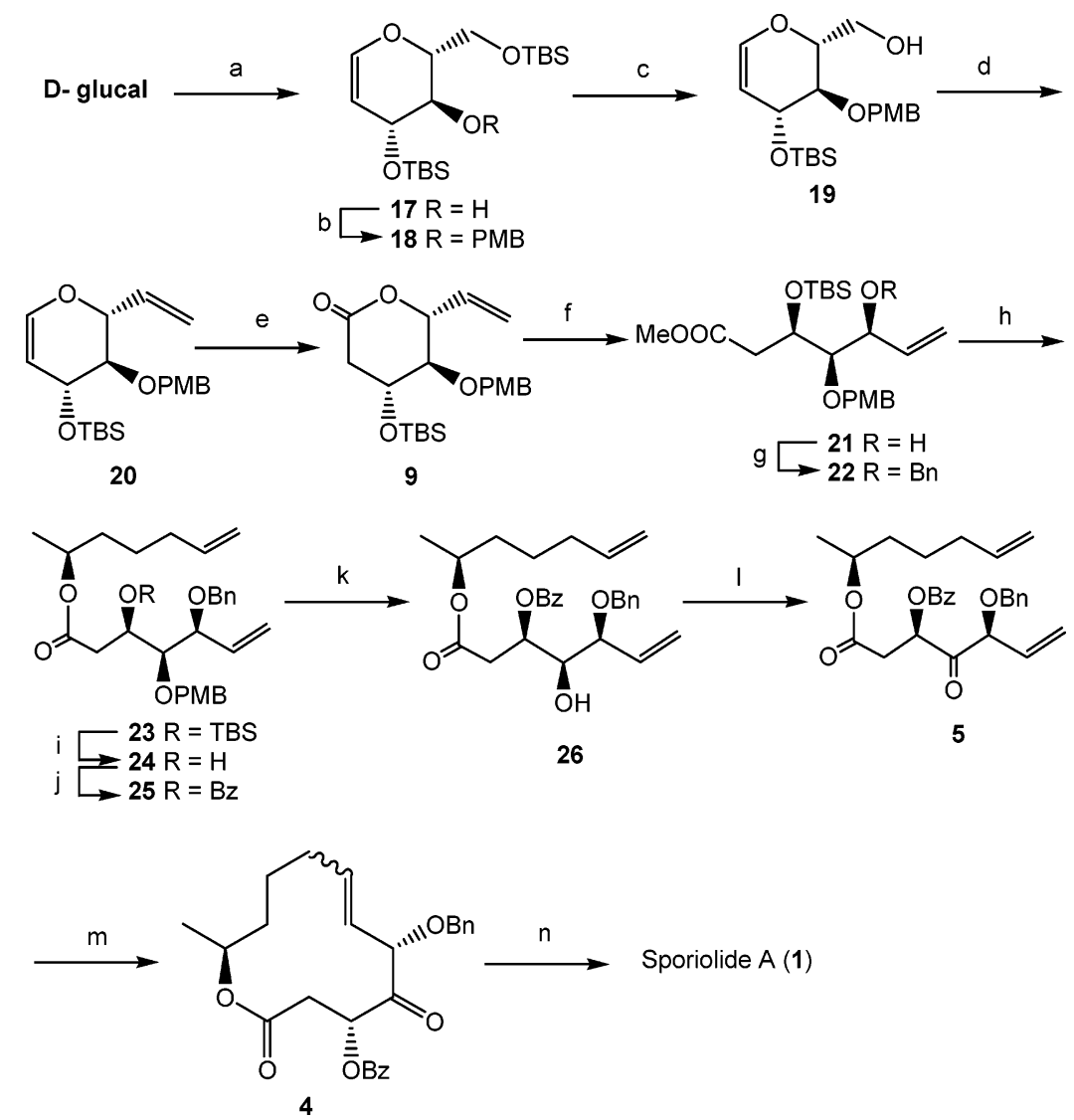

${ }^{a}$ Reagents and conditions: (a) ref 10, 90\%; (b) PMBCl, NaH, DMF, 2 h, 83\%; (c) HF/Pyr, THF, 2 h, 78\%; (d) (i) $\left(\mathrm{COCl}_{2}, \mathrm{DMSO}, \mathrm{TEA}, \mathrm{CH}_{2} \mathrm{Cl} 2,-78\right.$ ${ }^{\circ} \mathrm{C}$ to rt, $2 \mathrm{~h}$; (ii) $\mathrm{BuLi}, \mathrm{Ph}_{3} \mathrm{PCH}_{3} \mathrm{Br}$, THF, $-50{ }^{\circ} \mathrm{C}$ to rt, $3 \mathrm{~h}, 75 \%$ over two steps; (e) PCC, silica gel, $\mathrm{CH}_{2} \mathrm{Cl}_{2}, 45{ }^{\circ} \mathrm{C}, 6 \mathrm{~h}, 74 \%$; (f) $\mathrm{NaOMe}, \mathrm{MeOH}, 3 \mathrm{~h}$, 83\%; (g) BnOC(NH)CCl , TMSOTf, $\mathrm{CH}_{2} \mathrm{Cl}_{2}, 3 \mathrm{~h}, 75 \%$; (h) (i) LiOH, THF/MeOH/ $\mathrm{H}_{2} \mathrm{O}, 12 \mathrm{~h}$; (ii) (S)-6-hepten-2-ol, 2,4,6-trichlorobenzoyl chloride, TEA, DMAP, THF, 18 h, 79\% in two steps; (i) TBAF, THF, 4 h, 83\%; (j) BzCl, Pyr, DMAP, 4 h, 95\%; (k) DDQ, $\mathrm{CH}_{2} \mathrm{Cl}_{2} / \mathrm{H}_{2} \mathrm{O}, 2 \mathrm{~h}, 90 \%$; (l) Dess-Martin periodinane, $\mathrm{CH}_{2} \mathrm{Cl}_{2}, 3 \mathrm{~h}, 82 \%$; (m) 30\% $\mathrm{PhCH}=\mathrm{RuCl}_{2}\left(\mathrm{PCy}_{3}\right)_{2}, \mathrm{CH}_{2} \mathrm{Cl}_{2}$, reflux, $24 \mathrm{~h}, 72 \%$ (E/Z = 2/1); (n) $\mathrm{H}_{2}, \mathrm{Pd} / \mathrm{C}, \mathrm{MeOH}, 24 \mathrm{~h}, 91 \%$.

ate $\mathbf{5}$ in $\mathbf{5 8 \%}$ yield over four steps. Diene $\mathbf{5}$ was then exposed to the Grubbs catalyst $\left[\mathrm{PHCH}=\mathrm{RuCl}\left(\mathrm{PCy}_{3}\right)_{2}, 30 \mathrm{~mol} / \%, 1.4 \times\right.$ $10^{-4} \mathrm{M}$ in $\left.\mathrm{CH}_{2} \mathrm{Cl}_{2}\right]^{18}$ to undergo an intramolecular ring closure metathesis (RCM) to produce macrolide $\mathbf{4}$ in a form of $E, Z$ mixture $(E / Z=2 / 1)$. Final hydrogenation of $\mathbf{4}$ with $\mathrm{H}_{2}$ in the presence of $\mathrm{Pd} / \mathrm{C}$ reduced both benzyl and double bond to accomplish the total synthesis of sporiolide A, identical in all physical data to that reported for the natural product. ${ }^{3}$

In summary, we have finished the first total synthesis of sporiolide $\mathrm{A}$ in 16 steps and $6.1 \%$ overall yield. The required stereochemical configurations at $\mathrm{C}-3$ and $\mathrm{C}-5$ in sporiolide $\mathrm{A}$ were successfully secured by using D-glucal as the chiral template. PCC-promoted transformation of the cyclic enol ether to lactone, Yamaguchi esterification, and Grubbs ring closure metathesis greatly facilitated the synthesis of our target. As exemplified in this study, carbohydrate moieties provide versatile synthons in asymmetric synthesis of natural products. ${ }^{19}$ This report provides an attractive method for the preparation of other natural macrolides, such as pandangolide 1a, pandangolides 1

(18) Grubbs, R. H.; Chang, S. Tetrahedron 1998, 54, 4413-4450.

(19) (a) Boons, G.-J.; Hale, K. J. Organic Synthesis with Carbohydrates; Sheffield Academic Press: Sheffield, England, 2000. (b) Elliott, M. C.; Williams, E. J. Chem. Soc., Perkin Trans. 1 2001, 2303-2340. (c) Elliott, M. C. J. Chem. Soc., Perkin Trans. 1 2002, 2301-2323. (d) Harmange, J. C.; Figadere, B. Tetrahedron: Asymmetry 1993, 4, 1711-1754. (e) Ziegler, F. E.; Wang, Y. J. Org. Chem. 1998, 63, 426-427. (f) Carda, M.; Rodriguez, S.; Segovia, B.; Marco, J. A. J. Org. Chem. 2002, 67, 6560-6563. and 2, and sporiolide B. ${ }^{1 \mathrm{~d}, 20}$ The screening of cytotoxic activities toward other cell lines are currently under investigation and will be reported in due course.

\section{Experimental Section}

3,6-O-Di-tert-butyldimethylsilyl-4- $O$-p-methoxybenzyl-D-glucal (18). To a stirred solution of $\mathbf{1 7}(5.5 \mathrm{~g}, 14.7 \mathrm{mmol})$ in anhydrous DMF $(30 \mathrm{~mL})$ was added $\mathrm{NaH}(720 \mathrm{mg}, 30 \mathrm{mmol})$ portionwise at $0{ }^{\circ} \mathrm{C}$. After $30 \mathrm{~min}$, p-methoxybenzyl chloride $(2.4 \mathrm{~mL}, 18 \mathrm{mmol})$ was added dropwise under the same reaction conditions. The mixture was kept stirring at room temperature for $2 \mathrm{~h}$, poured into ice-water $(30 \mathrm{~mL})$, and extracted with EtOAc $(2 \times 100 \mathrm{~mL})$. The combined organic layer was dried over anhydrous $\mathrm{Na}_{2} \mathrm{SO}_{4}$ and concentrated to dryness. Purification of the residue on column chromatography (petroleum ether-EtOAc, 25:1) afforded $18(6.0 \mathrm{~g}, 83 \%)$ as a colorless syrup: $[\alpha]^{25}-20$ (c $\left.0.8, \mathrm{CHCl}_{3}\right) ;{ }^{1} \mathrm{H}$ NMR $\left(\mathrm{CDCl}_{3}\right) \delta 0.07(\mathrm{~s}, 6 \mathrm{H}), 0.10(\mathrm{~s}, 6 \mathrm{H})$, $0.90(\mathrm{~s}, 9 \mathrm{H}), 0.93(\mathrm{~s}, 9 \mathrm{H}), 3.62(\mathrm{t}, 1 \mathrm{H}, J=6.5 \mathrm{~Hz}), 3.80(\mathrm{~s}, 3 \mathrm{H})$, $3.84(\mathrm{~s}, 1 \mathrm{H}), 3.87(\mathrm{t}, 1 \mathrm{H}, J=6.6 \mathrm{~Hz}), 3.94(\mathrm{dd}, 1 \mathrm{H}, J=4.4,11.1$ $\mathrm{Hz}), 4.32(\mathrm{~d}, 1 \mathrm{H}, J=5.9 \mathrm{~Hz}), 4.62(\mathrm{dd}, 1 \mathrm{H}, J=2.4,8.3 \mathrm{~Hz}), 4.64$ $(\mathrm{d}, 1 \mathrm{H}, J=10.7 \mathrm{~Hz}), 4.75(\mathrm{~d}, 1 \mathrm{H}, J=10.8 \mathrm{~Hz}), 6.30(\mathrm{~d}, 1 \mathrm{H}, J=$ $6.1 \mathrm{~Hz}), 6.87(\mathrm{~d}, 2 \mathrm{H}, J=8.2 \mathrm{~Hz}), 7.26(\mathrm{~d}, 2 \mathrm{H}, J=8.2 \mathrm{~Hz}) ;{ }^{13} \mathrm{C}$

(20) (a) Smith, C. J.; Abbanat, D.; Bernan, V. S.; Maiese, W. M.; Greenstein, M.; Jompa, J.; Tahir, A.; Ireland, C. M. J. Nat. Prod. 2000, 63, 142-145. (b) Jadulco, R.; Proksch, P.; Wray, V.; Berg, A.; Grafe, U. J. Nat. Prod. 2001, 64, 527-530. 
$\operatorname{NMR}\left(\mathrm{CDCl}_{3}\right) \delta-5.4,-5.2,-4.6,-4.4,18.0,18.4,25.8,25.9$, 55.2, 61.9, 69.0, 73.5, 76.2, 78.0, 103.2, 113.8, 129.5, 130.6, 143.4, 159.2. Anal. Calcd for $\mathrm{C}_{26} \mathrm{H}_{46} \mathrm{O}_{5} \mathrm{Si}_{2}$ : C, 63.11; H, 9.37; Found: 63.39; H, 9.31.

3-O-tert-Butyldimethylsilyl-4-O-p-methoxybenzyl-D-glucal (19). To a solution of $\mathbf{1 8}(5.8 \mathrm{~g}, 11.7 \mathrm{mmol})$ in THF $(120 \mathrm{~mL})$ was added $\mathrm{HF} / \mathrm{Pyr}(70 \%, 7 \mathrm{~mL})$ dropwise at $0{ }^{\circ} \mathrm{C}$. The reaction mixture was warmed to room temperature, stirred for $2 \mathrm{~h}$, diluted with EtOAc $(100 \mathrm{~mL})$, and neutralized with saturated aqueous $\mathrm{NaHCO}_{3}$. The aqueous phase was further extracted with EtOAc $(100 \mathrm{~mL})$. After being washed with brine and dried over anhydrous $\mathrm{Na}_{2} \mathrm{SO}_{4}$, the organic layer was concentrated in vacuo. The residue was purified by column chromatography (petroleum ether-EtOAc, 4:1) to give $19(3.5 \mathrm{~g}, 78 \%)$ as a colorless syrup: $[\alpha]^{25} \mathrm{D}-18\left(c 0.6, \mathrm{CHCl}_{3}\right)$; ${ }^{1} \mathrm{H}$ NMR $\left(\mathrm{CDCl}_{3}\right) \delta 0.10(\mathrm{~s}, 6 \mathrm{H}), 0.92(\mathrm{~s}, 9 \mathrm{H}), 3.38(\mathrm{~d}, 1 \mathrm{H}, J=$ $7.7 \mathrm{~Hz}), 3.50(\mathrm{~d}, 1 \mathrm{H}, J=3.9 \mathrm{~Hz}), 3.81(\mathrm{~s}, 3 \mathrm{H}), 3.91(\mathrm{t}, 1 \mathrm{H}, J=$ $7.3 \mathrm{~Hz}), 4.62(\mathrm{~s}, 2 \mathrm{H}), 4.76(\mathrm{~d}, 1 \mathrm{H}, J=6.5 \mathrm{~Hz}), 5.56(\mathrm{~d}, 1 \mathrm{H}, J=$ $3.2 \mathrm{~Hz}), 5.82(\mathrm{dd}, 1 \mathrm{H}, J=4.1,9.7 \mathrm{~Hz}), 6.12(\mathrm{dd}, 1 \mathrm{H}, J=3.2,9.6$ $\mathrm{Hz}), 6.88(\mathrm{~d}, 2 \mathrm{H}, J=8.2 \mathrm{~Hz}), 7.29(\mathrm{~d}, 2 \mathrm{H}, J=8.2 \mathrm{~Hz}) ;{ }^{13} \mathrm{C} \mathrm{NMR}$ $\left(\mathrm{CDCl}_{3}\right) \delta-4.7,-4.4,17.9,25.8,55.2,61.9,68.6,73.5,76.4,77.0$, 103.7, 113.7, 129.6, 131.2, 143.4, 159.4. Anal. Calcd for $\mathrm{C}_{20} \mathrm{H}_{32} \mathrm{O}_{5^{-}}$ Si: C, 63.12; H, 8.48. Found: C, 63.50; H, 8.44.

$(2 R, 3 R, 4 R)-4$-(tert-Butyldimethylsilyloxy)-3-( $p$-methoxybenzyloxy)-2-vinyl-2,3-dihydro-2 $\boldsymbol{H}$-pyran (20). To a stirred solution of oxalyl chloride $(1.1 \mathrm{~mL}, 13 \mathrm{~mol})$ in dry $\mathrm{CH}_{2} \mathrm{Cl}_{2}(20 \mathrm{~mL})$ at -78 ${ }^{\circ} \mathrm{C}$ was added DMSO $(2.1 \mathrm{~mL}, 28 \mathrm{mmol})$ dropwise under $\mathrm{N}_{2}$ atmosphere. The reaction mixture was stirred under these conditions for $30 \mathrm{~min}$, and a solution of alcohol $19(3.4 \mathrm{~g}, 8.9 \mathrm{mmol})$ in dry $\mathrm{CH}_{2} \mathrm{Cl}_{2}(30 \mathrm{~mL})$ was then added slowly. After $1 \mathrm{~h}$ of stirring at $-78{ }^{\circ} \mathrm{C}, \mathrm{Et}_{3} \mathrm{~N}(8.3 \mathrm{~mL}, 60 \mathrm{~mol})$ was added, and the reaction was stirred for another $30 \mathrm{~min}$ at ambient temperature, quenched with saturated aqueous $\mathrm{NH}_{4} \mathrm{Cl}(30 \mathrm{~mL})$, and extracted with $\mathrm{CH}_{2} \mathrm{Cl}_{2}(30$ $\mathrm{mL})$. The combined organic phase was washed with water $(30 \mathrm{~mL})$ and brine $(30 \mathrm{~mL})$, dried over anhydrous $\mathrm{Na}_{2} \mathrm{SO}_{4}$, and concentrated under reduced pressure to furnish the aldehyde, which was directly used in the next step without further purification. In a parallel reaction flask, a $2.5 \mathrm{M}$ solution of $\mathrm{n}-\mathrm{BuLi}$ in hexane $(5.6 \mathrm{~mL}, 14$ mmol) was added under $\mathrm{N}_{2}$ atmosphere to a stirred suspension of methyltriphenylphosphonium bromide $(4.2 \mathrm{~g}, 11.6 \mathrm{mmol})$ in dry THF $(40 \mathrm{~mL})$ at $-50{ }^{\circ} \mathrm{C}$. The mixture was allowed to warm to room temperature, stirred for $1 \mathrm{~h}$, and cooled to $-50{ }^{\circ} \mathrm{C}$ again. To this mixture a solution of above crude aldehyde in dry THF (30 $\mathrm{mL}$ ) was added dropwise, and the resulting mixture was stirred at room temperature for $2 \mathrm{~h}$, quenched with aqueous $\mathrm{NH}_{4} \mathrm{Cl}$, and extracted with EtOAc $(2 \times 100 \mathrm{~mL})$. The combined organic extracts were dried over anhydrous $\mathrm{Na}_{2} \mathrm{SO}_{4}$ and concentrated in vacuo. The residue was purified by column chromatography (petroleum etherEtOAc, 10:1) to give 20 (2.5 g, 75\% yield over two steps) as a colorless syrup: $[\alpha]^{25} \mathrm{D}-18\left(\mathrm{c} 1, \mathrm{CHCl}_{3}\right) ;{ }^{1} \mathrm{H} \mathrm{NMR}\left(\mathrm{CDCl}_{3}\right) \delta$ $0.10(\mathrm{~s}, 6 \mathrm{H}), 0.92(\mathrm{~s}, 9 \mathrm{H}), 3.42(\mathrm{t}, 1 \mathrm{H}, J=6.5 \mathrm{~Hz}), 3.80(\mathrm{~s}, 3 \mathrm{H})$, $4.28(\mathrm{t}, 1 \mathrm{H}, J=7.5 \mathrm{~Hz}), 4.35(\mathrm{~d}, 1 \mathrm{H}, J=6.0 \mathrm{~Hz}), 4.58(\mathrm{~d}, 1 \mathrm{H}, J$ $=10.7 \mathrm{~Hz}), 4.66-4.68(\mathrm{~m}, 1 \mathrm{H}), 4.70(\mathrm{~d}, 1 \mathrm{H}, J=11.0 \mathrm{~Hz}), 5.26$ $(\mathrm{d}, 1 \mathrm{H}, J=10.5 \mathrm{~Hz}), 5.40(\mathrm{~d}, 1 \mathrm{H}, J=17.3 \mathrm{~Hz}), 5.97-6.06(\mathrm{~m}$, $1 \mathrm{H}), 6.32(\mathrm{~d}, 1 \mathrm{H}, J=6.0 \mathrm{~Hz}), 6.86(\mathrm{~d}, 2 \mathrm{H}, J=8.3 \mathrm{~Hz}), 7.26(\mathrm{~d}$, $2 \mathrm{H}, J=8.3 \mathrm{~Hz}) ;{ }^{13} \mathrm{C} \mathrm{NMR}\left(\mathrm{CDCl}_{3}\right) \delta-4.6,-4.5,18.0,25.8$, 55.2, 69.1, 73.8, 78.1, 80.1, 103.9, 113.7, 118.0, 129.6, 130.2, 134.6, 143.2, 159.2. Anal. Calcd for $\mathrm{C}_{21} \mathrm{H}_{32} \mathrm{O}_{4} \mathrm{Si}$ : C, 66.98; H, 8.57. Found: C, 67.23; H, 8.50.

(4R,5R,6R)-4-(tert-Butyldimethylsilyloxy)-5-( $p$-methoxybenzyloxy)-6-vinyltetrahydropyran-2-one (9). To a solution of compound 20 (840 mg, $2.2 \mathrm{mmol})$ in $\mathrm{CH}_{2} \mathrm{Cl}_{2}(100 \mathrm{~mL})$ was added the mixture of PCC $(1.5 \mathrm{~g}, 6.6 \mathrm{mmol})$ and silica gel $(1.8 \mathrm{~g})$. The stirred suspension was refluxed for $6 \mathrm{~h}$ and then cooled and filtered through Celite. The Celite pad was washed several times with EtOAc, and the combined filtrates were concentrated. The crude product was purified by column chromatography (petroleum etherEtOAc, 6:1) to give $9(650 \mathrm{mg}, 74 \%)$ as a colorless syrup: $[\alpha]^{25}$ $-14\left(c 0.8, \mathrm{CHCl}_{3}\right) ;{ }^{1} \mathrm{H} \mathrm{NMR}\left(\mathrm{CDCl}_{3}\right) \delta 0.08(\mathrm{~s}, 6 \mathrm{H}), 0.90(\mathrm{~s}$, 9H), $2.55(\mathrm{dd}, 1 \mathrm{H}, J=4.8,16.6 \mathrm{~Hz}), 2.89(\mathrm{dd}, 1 \mathrm{H}, J=4.2,16.6$
$\mathrm{Hz}), 3.46$ (t, $1 \mathrm{H}, J=4.8 \mathrm{~Hz}), 3.81(\mathrm{~s}, 3 \mathrm{H}), 4.17(\mathrm{dd}, 1 \mathrm{H}, J=4.3$, $8.6 \mathrm{~Hz}), 4.56(\mathrm{~d}, 1 \mathrm{H}, J=11.1 \mathrm{~Hz}), 4.62(\mathrm{~d}, 1 \mathrm{H}, J=11.1 \mathrm{~Hz})$, $4.66(\mathrm{t}, 1 \mathrm{H}, J=6.2 \mathrm{~Hz}), 5.25(\mathrm{~d}, 1 \mathrm{H}, J=10.5 \mathrm{~Hz}), 5.37(\mathrm{~d}, 1 \mathrm{H}$, $J=17.2 \mathrm{~Hz}), 5.90-5.98(\mathrm{~m}, 1 \mathrm{H}), 6.88(\mathrm{~d}, 2 \mathrm{H}, J=8.1 \mathrm{~Hz}), 7.23$ $(\mathrm{d}, 2 \mathrm{H}, J=8.1 \mathrm{~Hz}) ;{ }^{13} \mathrm{C} \mathrm{NMR}\left(\mathrm{CDCl}_{3}\right) \delta-4.9,-4.8,17.8,25.6$, 36.9, 55.3, 68.8, 72.7, 79.8, 81.0, 113.9, 117.8, 129.3, 129.5, 134.4, 159.5, 169.1. Anal. Calcd for $\mathrm{C}_{21} \mathrm{H}_{32} \mathrm{O}_{5} \mathrm{Si}$ : C, 64.25; H, 8.22. Found: C, 63.95; H, 8.18.

Methyl $(3 R, 4 R, 5 S)-3$-(tert-Butyldimethylsilyloxy)-5-hydroxy4-( $p$-methoxybenzyloxy)hept-6-enoate (21). To a solution of compound 9 (629 mg, $1.6 \mathrm{mmol})$ in anhydrous $\mathrm{MeOH}(15 \mathrm{~mL})$ was added $1 \mathrm{~N} \mathrm{NaOMe}$ in $\mathrm{MeOH}$ until $\mathrm{pH} 9-10$. The reaction mixture was stirred at room temperature for $3 \mathrm{~h}$ under these conditions, neutralized with Amberlite IR-120 $\left(\mathrm{H}^{+}\right)$, and filtered. The filtrate was concentrated to dryness under diminished pressure and purified by silica gel column chromatography (petroleum ether-EtOAc, 5:1) to give $21(550 \mathrm{mg}, 83 \%)$ as a colorless syrup: $[\alpha]^{25} \mathrm{D}-10\left(\mathrm{c} 1, \mathrm{CHCl}_{3}\right) ;{ }^{1} \mathrm{H} \mathrm{NMR}\left(\mathrm{CDCl}_{3}\right) \delta 0.08(\mathrm{~s}, 6 \mathrm{H}), 0.88$ (s, 9H), $2.56(\mathrm{dd}, 1 \mathrm{H}, J=5.0,16.5 \mathrm{~Hz}), 2.89(\mathrm{dd}, 1 \mathrm{H}, J=4.3$, $16.5 \mathrm{~Hz}), 3.46(\mathrm{t}, 1 \mathrm{H}, J=5.1 \mathrm{~Hz}), 3.49(\mathrm{~s}, 3 \mathrm{H}), 3.81(\mathrm{~s}, 3 \mathrm{H}), 4.17$ $(\mathrm{dd}, 1 \mathrm{H}, J=4.4,8.7 \mathrm{~Hz}), 4.56(\mathrm{~d}, 1 \mathrm{H}, J=11.1 \mathrm{~Hz}), 4.62(\mathrm{~d}, 1 \mathrm{H}$, $J=11.1 \mathrm{~Hz}), 4.66(\mathrm{t}, 1 \mathrm{H}, J=6.3 \mathrm{~Hz}), 5.25(\mathrm{~d}, 1 \mathrm{H}, J=10.6 \mathrm{~Hz})$, $5.37(\mathrm{~d}, 1 \mathrm{H}, J=17.2 \mathrm{~Hz}), 5.90-5.98(\mathrm{~m}, 1 \mathrm{H}), 6.88(\mathrm{~d}, 2 \mathrm{H}, J=$ $8.2 \mathrm{~Hz}), 7.23(\mathrm{~d}, 2 \mathrm{H}, J=8.2 \mathrm{~Hz}) ;{ }^{13} \mathrm{C} \mathrm{NMR}\left(\mathrm{CDCl}_{3}\right) \delta-4.8$, $-4.8,17.9,25.6,37.0,51.6,55.3,68.8,72.7,79.8,81.0,113.8$, $117.9,129.5,129.7,134.4,159.6,172.2$. Anal. Calcd for $\mathrm{C}_{22} \mathrm{H}_{36} \mathrm{O}_{6}$ Si: C, 62.23; H, 8.55. Found: C, 62.01; H, 8.64.

Methyl (3R,4R,5S)-5-Benzyloxy-3-(tert-butyldimethylsilyloxy)4-(p-methoxybenzyloxy)hept-6-enoate (22). To a solution of 21 (509 $\mathrm{mg}, 1.2 \mathrm{mmol}$ ) and benzyltrichloroacetimidate $(460 \mathrm{mg}, 1.8$ mmol) in dry $\mathrm{CH}_{2} \mathrm{Cl}_{2}(15 \mathrm{~mL})$ at $0{ }^{\circ} \mathrm{C}$ was added TMSOTf $(9.0$ $\mu \mathrm{L}, 0.02 \mathrm{mmol})$ under $\mathrm{N}_{2}$ protection. The reaction mixture was monitored by TLC until all starting material was consumed and then quenched with $\mathrm{Et}_{3} \mathrm{~N}$ and concentrated to dryness. The residue was subjected to the silica gel column chromatography (petroleum ether-EtOAc, 6:1) to give $\mathbf{2 2}$ (463 $\mathrm{mg}, 75 \%)$ as a colorless syrup: $[\alpha]^{25} \mathrm{D}-12\left(c 0.5, \mathrm{CHCl}_{3}\right) ;{ }^{1} \mathrm{H}$ NMR $\left(\mathrm{CDCl}_{3}\right) \delta 0.09(\mathrm{~s}, 6 \mathrm{H}), 0.86$ $(\mathrm{s}, 9 \mathrm{H}), 2.42(\mathrm{dd}, 1 \mathrm{H}, J=8.8,15.1 \mathrm{~Hz}), 2.63(\mathrm{dd}, 1 \mathrm{H}, J=2.9$, $15.3 \mathrm{~Hz}), 3.62(\mathrm{~s}, 3 \mathrm{H}), 3.68-3.70(\mathrm{~m}, 1 \mathrm{H}), 3.80(\mathrm{~s}, 3 \mathrm{H}), 4.10(\mathrm{~d}$, $1 \mathrm{H}, J=9.6 \mathrm{~Hz}), 4.26-4.32(\mathrm{~m}, 2 \mathrm{H}), 4.56(\mathrm{~d}, 1 \mathrm{H}, J=11.3 \mathrm{~Hz})$, $4.58(\mathrm{~d}, 1 \mathrm{H}, J=12.1 \mathrm{~Hz}), 4.73(\mathrm{~d}, 1 \mathrm{H}, J=11.3 \mathrm{~Hz}), 5.33(\mathrm{~d}, 1 \mathrm{H}$, $J=17.1 \mathrm{~Hz}), 5.38(\mathrm{~d}, 1 \mathrm{H}, J=10.4 \mathrm{~Hz}), 5.95-6.04(\mathrm{~m}, 1 \mathrm{H}), 6.85$ $(\mathrm{d}, 2 \mathrm{H}, J=8.0 \mathrm{~Hz}), 7.25-7.40(\mathrm{~m}, 7 \mathrm{H}) ;{ }^{13} \mathrm{C} \mathrm{NMR}\left(\mathrm{CDCl}_{3}\right) \delta$ $-5.1,-4.7,17.9,25.7,38.3,51.4,55.2,69.7,69.7,72.9,80.2$, 82.2, 113.6, 119.8, 127.3, 127.5, 128.2, 129.4, 130.8, 135.6, 138.7, 159.1, 172.6. Anal. Calcd for $\mathrm{C}_{29} \mathrm{H}_{42} \mathrm{O}_{6} \mathrm{Si}$ : C, 67.67; H, 8.22. Found: C, 67.91; H, 8.13.

$(S)$-Hept-6-en-2-yl $\quad(3 R, 4 R, 5 S)$-5-Benzyloxy-3-(tert-butyldimethylsilyloxy)-4-( $p$-methoxybenzyloxy)hept-6-enoate (23). The methyl ester 22 (437 mg, $0.85 \mathrm{mmol}$ ) was dissolved in THF/MeOH/ $\mathrm{H}_{2} \mathrm{O}(15 \mathrm{~mL}, 2: 1: 1)$ and cooled with ice-water bath. $\mathrm{LiOH} \cdot \mathrm{H}_{2} \mathrm{O}$ (105 $\mathrm{mg}, 2.5 \mathrm{mmol}$ ) was added and the resulting mixture was stirred at room temperature for $12 \mathrm{~h}$, neutralized with $1 \mathrm{~N} \mathrm{HCl}$, and then extracted with EtOAc. The combined organic phase was dried over anhydrous $\mathrm{Na}_{2} \mathrm{SO}_{4}$ and concentrated to afford acid intermediate. Without further purification, the crude acid was dissolved in anhydrous THF $(15 \mathrm{~mL})$ and treated with triethylamine $(0.12 \mathrm{~mL}$, $0.9 \mathrm{mmol})$ and 2,4,6-trichlorobenzoyl chloride $(0.13 \mathrm{~mL}, 0.85$ $\mathrm{mmol}$ ), successively. After being stirred at room temperature for 1 $\mathrm{h}$, a solution of $(S)$-6-hepten-2-ol (113 $\mathrm{mg}, 1.0 \mathrm{mmol})$ and DMAP $(110 \mathrm{mg}, 0.9 \mathrm{mmol})$ in THF $(5 \mathrm{~mL})$ were added. The reaction mixture was stirred at room temperature for another $18 \mathrm{~h}$ and was then quenched by aqueous $\mathrm{NH}_{4} \mathrm{Cl}$. The water phase was separated and extracted with EtOAc $(2 \times 25 \mathrm{~mL})$. The combined organic extracts were dried and evaporated. Purification of the residue by silica gel column chromatography (petroleum ether-EtOAc, 7:1) gave 23 (400 mg, 79\% over two steps) as a colorless syrup: $[\alpha]^{25} \mathrm{D}$ $-17\left(c \quad 0.5, \mathrm{CHCl}_{3}\right) ;{ }^{1} \mathrm{H}$ NMR $\left(\mathrm{CDCl}_{3}\right) \delta 0.06(\mathrm{~s}, 3 \mathrm{H}), 0.08(\mathrm{~s}$, $3 \mathrm{H}), 0.87(\mathrm{~s}, 9 \mathrm{H}), 1.23(\mathrm{~d}, 3 \mathrm{H}, J=6.2 \mathrm{~Hz}), 1.36-1.56(\mathrm{~m}, 3 \mathrm{H})$, 
$1.60-1.63(\mathrm{~m}, 1 \mathrm{H}), 2.09(\mathrm{dd}, 2 \mathrm{H}, J=6.8,13.4 \mathrm{~Hz}), 2.43(\mathrm{dd}, 1 \mathrm{H}$, $J=8.3,15.8 \mathrm{~Hz}), 2.69(\mathrm{dd}, 1 \mathrm{H}, J=3.6,15.8 \mathrm{~Hz}), 3.77(\mathrm{dd}, 1 \mathrm{H}$, $J=3.9,4.8 \mathrm{~Hz}), 3.86(\mathrm{~s}, 3 \mathrm{H}), 4.16(\mathrm{dd}, 1 \mathrm{H}, J=2.6,8.3 \mathrm{~Hz})$, $4.36-4.39(\mathrm{~m}, 2 \mathrm{H}), 4.62(\mathrm{~d}, 1 \mathrm{H}, J=10.9 \mathrm{~Hz}), 4.65(\mathrm{~d}, 1 \mathrm{H}, J=$ $11.1 \mathrm{~Hz}), 4.78(\mathrm{~d}, 1 \mathrm{H}, J=11.1 \mathrm{~Hz}), 4.90-4.95(\mathrm{~m}, 1 \mathrm{H}), 5.01(\mathrm{~d}$, $1 \mathrm{H}, J=10.2 \mathrm{~Hz}), 5.05(\mathrm{~d}, 1 \mathrm{H}, J=18.4 \mathrm{~Hz}), 5.40(\mathrm{~d}, 1 \mathrm{H}, J=$ $17.5 \mathrm{~Hz}), 5.43(\mathrm{~d}, 1 \mathrm{H}, J=10.7 \mathrm{~Hz}), 5.80-5.86(\mathrm{~m}, 1 \mathrm{H}), 6.01-$ $6.10(\mathrm{~m}, 1 \mathrm{H}), 6.91(\mathrm{~d}, 2 \mathrm{H}, J=8.4 \mathrm{~Hz}), 7.26-7.39(\mathrm{~m}, 7 \mathrm{H}) ;{ }^{13} \mathrm{C}$ NMR $\left(\mathrm{CDCl}_{3}\right) \delta-4.9,-4.7,17.9,19.8,24.6,25.8,33.5,35.3$, $38.7,55.2,69.3,69.7,70.8,72.9,80.2,82.2,113.6,114.7,119.7$, 127.2, 127.4, 128.2, 129.4, 130.8, 135.6, 138.4, 138.8, 159.0, 171.7 . Anal. Calcd for $\mathrm{C}_{35} \mathrm{H}_{52} \mathrm{O}_{6} \mathrm{Si}$ : C, 70.43; H, 8.78. Found: C, 70.72; H, 8.91.

(S)-Hept-6-en-2-yl $\quad(3 R, 4 S, 5 S)$-5-Benzyloxy-3-hydroxy-4-( $p$ methoxybenzyloxy)hept-6-enoate (24). To a solution of silyl ether 23 (358 $\mathrm{mg}, 0.6 \mathrm{mmol})$ in THF $(10 \mathrm{~mL})$ was added TBAF $(1.2$ $\mathrm{mL}$ of $1 \mathrm{M}$ solution in THF, $1.2 \mathrm{mmol}$ ) at $0{ }^{\circ} \mathrm{C}$. The mixture was stirred under these conditions for $30 \mathrm{~min}$, followed by an additional $4 \mathrm{~h}$ of stirring at room temperature. At the end of which time TLC indicated the reaction complete. Then the reaction mixture was concentrated under vacuum and the residue was subjected to column chromatography (petroleum ether-EtOAc, 4:1) to furnish $\mathbf{2 4}$ (240 $\mathrm{mg}, 83 \%)$ as a colorless syrup: $[\alpha]^{25} \mathrm{D}-18\left(\mathrm{c} 1, \mathrm{CHCl}_{3}\right) ;{ }^{1} \mathrm{H} \mathrm{NMR}$ $\left(\mathrm{CDCl}_{3}\right) \delta 1.18(\mathrm{~d}, 3 \mathrm{H}, J=6.2 \mathrm{~Hz}), 1.36-1.55(\mathrm{~m}, 3 \mathrm{H}), 1.57-$ $1.60(\mathrm{~m}, 1 \mathrm{H}), 2.04(\mathrm{dd}, 2 \mathrm{H}, J=7.0,13.8 \mathrm{~Hz}), 2.43-2.50(\mathrm{~m}, 2 \mathrm{H})$, 3.09 (br s, 1H), 3.39-3.41 (m, 1H), $3.80(\mathrm{~s}, 3 \mathrm{H}), 4.08$ (t, $1 \mathrm{H}, J=$ $6.5 \mathrm{~Hz}), 4.32$ (br s, 1H), $4.40(\mathrm{~d}, 1 \mathrm{H}, J=11.4 \mathrm{~Hz}), 4.44(\mathrm{~d}, 1 \mathrm{H}$, $J=11.4 \mathrm{~Hz}), 4.60(\mathrm{~d}, 1 \mathrm{H}, J=11.9 \mathrm{~Hz}), 4.63(\mathrm{~d}, 1 \mathrm{H}, J=11.9$ $\mathrm{Hz}), 4.89-4.96(\mathrm{~m}, 2 \mathrm{H}), 4.99(\mathrm{~d}, 1 \mathrm{H}, J=17.5 \mathrm{~Hz}), 5.39$ (d, 1H, $J=16.6 \mathrm{~Hz}), 5.41(\mathrm{~d}, 1 \mathrm{H}, J=10.8 \mathrm{~Hz}), 5.74-5.80(\mathrm{~m}, 1 \mathrm{H}), 5.81-$ $5.91(\mathrm{~m}, 1 \mathrm{H}), 6.85(\mathrm{~d}, 2 \mathrm{H}, J=8.3 \mathrm{~Hz}), 7.20-7.34(\mathrm{~m}, 7 \mathrm{H}) ;{ }^{13} \mathrm{C}$ $\operatorname{NMR}\left(\mathrm{CDCl}_{3}\right) \delta 19.9,24.6,33.4,35.3,38.9,55.2,68.1,70.7,71.0$ $73.2,80.4,81.1,113.7,114.7,119.5,127.7,127.9,128.4,129.8$, 135.6, 137.7, 138.4, 159.3, 171.4. Anal. Calcd for $\mathrm{C}_{29} \mathrm{H}_{38} \mathrm{O}_{6}$ : C, 72.17; H, 7.94. Found: C, 71.90; H, 8.05.

$(S)$-Hept-6-en-2-yl $(3 R, 4 S, 5 S)$-3-Benzoyloxy-5-benzyloxy-4-( $p$ methoxybenzyloxy)hept-6-enoate (25). To the solution of $\mathbf{2 4}$ (216 $\mathrm{mg}, 0.45 \mathrm{mmol})$ in pyridine $(5 \mathrm{~mL})$ were added benzoyl chloride $(0.06 \mathrm{~mL}, 0.67 \mathrm{mmol})$ and a catalytic amount of DMAP at $0{ }^{\circ} \mathrm{C}$. The mixture was stirred at room temperature for $3 \mathrm{~h}$ and coevaporated with toluene under diminished pressure. Purification of the residue on silica gel column chromatography (petroleum etherEtOAc, 8:1) afforded $25(250 \mathrm{mg}, 95 \%)$ as a colorless syrup: $[\alpha]^{25} \mathrm{D}$ $-17\left(c 0.5, \mathrm{CHCl}_{3}\right) ;{ }^{1} \mathrm{H} \mathrm{NMR}\left(\mathrm{CDCl}_{3}\right) \delta 1.12(\mathrm{~d}, 3 \mathrm{H}, J=6.1 \mathrm{~Hz})$, $1.29-1.45(\mathrm{~m}, 3 \mathrm{H}), 1.50-1.54(\mathrm{~m}, 1 \mathrm{H}), 1.97(\mathrm{dd}, 2 \mathrm{H}, J=7.2$, $14.0 \mathrm{~Hz}), 2.72-2.85(\mathrm{~m}, 2 \mathrm{H}), 3.79(\mathrm{~s}, 3 \mathrm{H}), 3.81(\mathrm{dd}, 1 \mathrm{H}, J=3.6$, $5.8 \mathrm{~Hz}), 3.96(\mathrm{t}, 1 \mathrm{H}, J=6.7 \mathrm{~Hz}), 4.28(\mathrm{~d}, 1 \mathrm{H}, J=11.4 \mathrm{~Hz}), 4.53$ $(\mathrm{d}, 1 \mathrm{H}, J=10.9 \mathrm{~Hz}), 4.56(\mathrm{~d}, 1 \mathrm{H}, J=10.3 \mathrm{~Hz}), 4.68(\mathrm{~d}, 1 \mathrm{H}, J=$ $10.9 \mathrm{~Hz}), 4.86(\mathrm{t}, 1 \mathrm{H}, J=6.1 \mathrm{~Hz}), 4.91(\mathrm{~d}, 1 \mathrm{H}, J=11.4 \mathrm{~Hz}), 4.95$ $(\mathrm{d}, 1 \mathrm{H}, J=19.1 \mathrm{~Hz}), 5.35(\mathrm{~d}, 1 \mathrm{H}, J=11.4 \mathrm{~Hz}), 5.36(\mathrm{~d}, 1 \mathrm{H}, J=$ $16.1 \mathrm{~Hz}), 5.67-5.74(\mathrm{~m}, 1 \mathrm{H}), 5.76-5.81(\mathrm{~m}, 1 \mathrm{H}), 5.87-5.96(\mathrm{~m}$, $1 \mathrm{H}), 6.83(\mathrm{~d}, 2 \mathrm{H}, J=8.3 \mathrm{~Hz}), 7.18-7.29(\mathrm{~m}, 7 \mathrm{H}), 7.38(\mathrm{t}, 2 \mathrm{H}, J$ $=7.6 \mathrm{~Hz}), 7.53(\mathrm{t}, 1 \mathrm{H}, J=7.1 \mathrm{~Hz}), 7.97(\mathrm{~d}, 2 \mathrm{H}, J=7.7 \mathrm{~Hz}) ;{ }^{13} \mathrm{C}$ $\operatorname{NMR}\left(\mathrm{CDCl}_{3}\right) \delta 19.7,24.5,33.4,35.2,36.3,55.2,70.2,70.5,71.3$, $73.8,80.2,80.4,113.6,114.7,119.7,127.4,127.9,128.2,129.7$, 129.8, 130.1, 130.1, 132.8, 135.6, 138.0, 138.3, 159.2, 165.6, 170.1. Anal. Calcd for $\mathrm{C}_{36} \mathrm{H}_{42} \mathrm{O}_{7}$ : C, 73.70; H, 7.22. Found: C, 73.43; H, 7.13 .

(S)-Hept-6-en-2-yl (3R,4S,5S)-3-Benzoyloxy-5-benzyloxy-4hydroxyhept-6-enoate (26). A solution of ester $\mathbf{2 5}$ (224 mg, 0.38 $\mathrm{mmol})$ in $\mathrm{CH}_{2} \mathrm{Cl}_{2}(10 \mathrm{~mL})$ at $0{ }^{\circ} \mathrm{C}$ was treated with DDQ (173 mg, $0.76 \mathrm{mmol})$ in the presence of water $(1 \mathrm{~mL})$. The mixture was stirred at room temperature for $2 \mathrm{~h}$ and then quenched with saturated aqueous $\mathrm{NaHCO}_{3}$. The aqueous layer was separated and extracted with $\mathrm{CH}_{2} \mathrm{Cl}_{2}(2 \times 20 \mathrm{~mL})$. The combined organic extracts were dried over anhydrous $\mathrm{Na}_{2} \mathrm{SO} 4$ and concentrated to dryness. Column chromatography of the residue (petroleum ether-EtOAc, 5:1) gave $26(160 \mathrm{mg}, 90 \%)$ as a colorless syrup: $[\alpha]^{25} \mathrm{D}-17\left(c 0.8, \mathrm{CHCl}_{3}\right)$; ${ }^{1} \mathrm{H} \mathrm{NMR}\left(\mathrm{CDCl}_{3}\right) \delta 1.13(\mathrm{~d}, 3 \mathrm{H}, J=6.1 \mathrm{~Hz}), 1.29-1.47(\mathrm{~m}, 3 \mathrm{H})$, $1.50-1.54(\mathrm{~m}, 1 \mathrm{H}), 1.97(\mathrm{dd}, 2 \mathrm{H}, J=6.9,13.4 \mathrm{~Hz}), 2.53$ (br s, $1 \mathrm{H}), 2.87(\mathrm{~d}, 2 \mathrm{H}, J=6.5 \mathrm{~Hz}), 3.83-3.89(\mathrm{~m}, 2 \mathrm{H}), 4.27(\mathrm{~d}, 1 \mathrm{H}, J$ $=11.2 \mathrm{~Hz}), 4.52(\mathrm{~d}, 1 \mathrm{H}, J=11.2 \mathrm{~Hz}), 4.87(\mathrm{t}, 1 \mathrm{H}, J=6.0 \mathrm{~Hz})$, $4.92(\mathrm{~d}, 1 \mathrm{H}, J=11.6 \mathrm{~Hz}), 4.95(\mathrm{~d}, 1 \mathrm{H}, J=18.7 \mathrm{~Hz}), 5.36(\mathrm{~d}, 1 \mathrm{H}$, $J=17.4 \mathrm{~Hz}), 5.41(\mathrm{~d}, 1 \mathrm{H}, J=10.3 \mathrm{~Hz}), 5.68-5.75(\mathrm{~m}, 1 \mathrm{H}), 5.80$ $(\mathrm{t}, 1 \mathrm{H}, J=6.3 \mathrm{~Hz}), 5.85-5.94(\mathrm{~m}, 1 \mathrm{H}), 7.14-7.24(\mathrm{~m}, 5 \mathrm{H}), 7.39$ $(\mathrm{t}, 2 \mathrm{H}, J=7.4 \mathrm{~Hz}), 7.54(\mathrm{t}, 1 \mathrm{H}, J=7.0 \mathrm{~Hz}), 7.97$ (d, 2H, $J=7.9$ $\mathrm{Hz}) ;{ }^{13} \mathrm{C} \mathrm{NMR}\left(\mathrm{CDCl}_{3}\right) \delta 19.8,24.5,33.4,35.2,36.8,69.6,70.7$, 71.5, 73.8, 81.0, 114.7, 120.7, 127.6, 128.1, 128.2, 128.3, 129.7, $130.1,132.9,135.2,137.6,138.4,165.5,170.2$. Anal. Calcd for $\mathrm{C}_{28} \mathrm{H}_{34} \mathrm{O}_{6}$ : C, 72.08; H, 7.35. Found: C, 72.42; H, 7.26.

(S)-Hept-6-en-2-yl (3R,5S)-3-Benzoyloxy-5-benzyloxy-4-oxohept-6-enoate (5). To a solution of $\mathbf{2 6}(149 \mathrm{mg}, 0.32 \mathrm{mmol})$ in dry $\mathrm{CH}_{2} \mathrm{Cl}_{2}(15 \mathrm{~mL})$ was added Dess - Martin periodinane (203 mg, $0.48 \mathrm{mmol}$ ) and the reaction mixture was stirred for $3 \mathrm{~h}$ at ambient temperature. After extraction with saturated aqueous $\mathrm{NaHCO}_{3}$, the organic layer was separated, dried over $\mathrm{Na}_{2} \mathrm{SO}_{4}$, and concentrated under reduced pressure. The remaining residue was subjected to column chromatography on silica gel (petroleum ether-EtOAc, 9:1) to furnish compound $\mathbf{5}(121 \mathrm{mg}, 82 \%)$ as a colorless syrup: $[\alpha]^{25} \mathrm{D}$ $-17\left(c 1, \mathrm{CHCl}_{3}\right),{ }^{1} \mathrm{H} \mathrm{NMR}\left(\mathrm{CDCl}_{3}\right) \delta 1.15(\mathrm{~d}, 3 \mathrm{H}, J=6.2 \mathrm{~Hz})$, $1.33-1.48(\mathrm{~m}, 3 \mathrm{H}), 1.51-1.55(\mathrm{~m}, 1 \mathrm{H}), 2.01(\mathrm{dd}, 2 \mathrm{H}, J=7.2$, $14.1 \mathrm{~Hz}), 2.82(\mathrm{dd}, 1 \mathrm{H}, J=9.0,16.2 \mathrm{~Hz}), 3.07(\mathrm{dd}, 1 \mathrm{H}, J=3.5$, $16.1 \mathrm{~Hz}), 4.64(\mathrm{~d}, 1 \mathrm{H}, J=5.8 \mathrm{~Hz}), 4.66(\mathrm{~d}, 1 \mathrm{H}, J=10.8 \mathrm{~Hz})$, $4.71(\mathrm{~d}, 1 \mathrm{H}, J=11.6 \mathrm{~Hz}), 4.91-4.96(\mathrm{~m}, 2 \mathrm{H}), 4.98(\mathrm{~d}, 1 \mathrm{H}, J=$ $17.7 \mathrm{~Hz}), 5.42$ (d, $1 \mathrm{H}, J=10.5 \mathrm{~Hz}), 5.49(\mathrm{~d}, 1 \mathrm{H}, J=17.2 \mathrm{~Hz})$, $5.65-5.77(\mathrm{~m}, 1 \mathrm{H}), 5.84-5.92(\mathrm{~m}, 1 \mathrm{H}), 6.08(\mathrm{dd}, 1 \mathrm{H}, J=3.4,9.0$ $\mathrm{Hz}), 7.28-7.37(\mathrm{~m}, 5 \mathrm{H}), 7.43(\mathrm{t}, 2 \mathrm{H}, J=7.6 \mathrm{~Hz}), 7.57(\mathrm{t}, 1 \mathrm{H}, J$ $=7.4 \mathrm{~Hz}), 8.01(\mathrm{~d}, 2 \mathrm{H}, J=7.7 \mathrm{~Hz}) ;{ }^{13} \mathrm{C} \mathrm{NMR}\left(\mathrm{CDCl}_{3}\right) \delta 19.8$, 24.6, 33.4, 35.2, 35.8, 71.9, 72.1, 73.3, 84.1, 114.8, 119.8, 127.9, 128.0, 128.4, 128.5, 129.1, 129.8, 132.3, 133.4, 137.0, 138.3, 165.5, 168.8, 203.1. Anal. Calcd for $\mathrm{C}_{28} \mathrm{H}_{32} \mathrm{O}_{6}$ : C, 72.39; H, 6.94. Found: C, 72.04; H, 7.05.

Lactone (4). Diene 5 (97 mg, $0.21 \mathrm{mmol}$ ) was dissolved in dry, degassed $\mathrm{CH}_{2} \mathrm{Cl}_{2}(10 \mathrm{~mL})$. This mixture was added dropwise within $1 \mathrm{~h}$ to a refluxing solution of ruthenium catalyst $(54 \mathrm{mg}, 0.06 \mathrm{mmol}$ ) in dry, degassed $\mathrm{CH}_{2} \mathrm{Cl}_{2}(470 \mathrm{~mL})$. The reaction mixture was stirred under these conditions until the complete consumption of $\mathbf{5}$ (20$24 \mathrm{~h}$, TLC monitoring). After removing solvent in vacuo, the residue was purified by silica gel column chromatography (petroleum ether-EtOAc, 9:1) to yield $4(66 \mathrm{mg}, 72 \%)$ as a mixture of $Z, E-$ isomers $(Z / E=1 / 2)$ : selected ${ }^{1} \mathrm{H} \mathrm{NMR}\left(\mathrm{CDCl}_{3}\right)$ for $E$-isomer, $\delta$ $1.18(\mathrm{~d}, 3 \mathrm{H}, J=6.5 \mathrm{~Hz}), 1.40-1.49(\mathrm{~m}, 1 \mathrm{H}), 1.56-1.76(\mathrm{~m}, 3 \mathrm{H})$, 1.84 (dd, $1 \mathrm{H}, J=11.2,23.8 \mathrm{~Hz}), 2.30-2.33(\mathrm{~m}, 1 \mathrm{H}), 2.97$ (dd, $1 \mathrm{H}, J=5.0,17.9 \mathrm{~Hz}), 3.47(\mathrm{dd}, 1 \mathrm{H}, J=3.3,17.8 \mathrm{~Hz}), 4.57(\mathrm{~d}$, $1 \mathrm{H}, J=7.9 \mathrm{~Hz}), 4.68(\mathrm{~s}, 2 \mathrm{H}), 4.95-4.97(\mathrm{~m}, 1 \mathrm{H}), 5.41(\mathrm{dd}, 1 \mathrm{H}$, $J=3.9,15.7 \mathrm{~Hz}), 5.84-5.91(\mathrm{~m}, 1 \mathrm{H}), 5.96(\mathrm{t}, 1 \mathrm{H}, J=3.8 \mathrm{~Hz})$, $7.16-7.21(\mathrm{~m}, 2 \mathrm{H}), 7.32-7.36(\mathrm{~m}, 3 \mathrm{H}), 7.44(\mathrm{t}, 3 \mathrm{H}, J=7.2 \mathrm{~Hz})$, $8.12(\mathrm{~d}, 2 \mathrm{H}, J=7.9 \mathrm{~Hz})$; for Z-isomer, $\delta 1.24(\mathrm{~d}, 3 \mathrm{H}, J=6.3 \mathrm{~Hz})$, $1.40-1.49(\mathrm{~m}, 1 \mathrm{H}), 1.56-1.76(\mathrm{~m}, 3 \mathrm{H}), 2.07-2.12(\mathrm{~m}, 1 \mathrm{H}), 2.14-$ $2.19(\mathrm{~m}, 1 \mathrm{H}), 2.83(\mathrm{dd}, 1 \mathrm{H}, J=9.8,15.0 \mathrm{~Hz}), 3.06(\mathrm{dd}, 1 \mathrm{H}, J=$ $2.9,15.0 \mathrm{~Hz}), 4.42(\mathrm{~d}, 1 \mathrm{H}, J=11.6 \mathrm{~Hz}), 4.58(\mathrm{~d}, 1 \mathrm{H}, J=11.8$ $\mathrm{Hz}), 4.73(\mathrm{~d}, 1 \mathrm{H}, J=8.3 \mathrm{~Hz}), 4.95-4.97(\mathrm{~m}, 1 \mathrm{H}), 5.69(\mathrm{t}, 1 \mathrm{H}, J$ $=8.6 \mathrm{~Hz}), 5.89-5.93(\mathrm{~m}, 1 \mathrm{H}), 6.10(\mathrm{dd}, 1 \mathrm{H}, J=3.0,9.8 \mathrm{~Hz})$, $7.30-7.33(\mathrm{~m}, 2 \mathrm{H}), 7.34-7.37(\mathrm{~m}, 3 \mathrm{H}), 7.57(\mathrm{t}, 3 \mathrm{H}, J=7.8 \mathrm{~Hz})$, $8.02(\mathrm{~d}, 2 \mathrm{H}, J=7.9 \mathrm{~Hz})$; selected ${ }^{13} \mathrm{C} \mathrm{NMR}\left(\mathrm{CDCl}_{3}\right)$ for $E$-isomer, $\delta 18.3,20.3,29.7,32.3,35.3,71.2,72.4,72.5,84.2,124.4,127.5$, $128.1,128.4,128.6,129.6,129.9,130.0,132.5,133.3,137.1,165.7$, 168.8, 200.9; $\mathrm{ESI}(+)-\mathrm{MS}$ calcd for $\mathrm{C}_{26} \mathrm{H}_{28} \mathrm{O}_{6} 436.19$ [M], found $437.5[\mathrm{M}+\mathrm{H}]^{+}, 459.5[\mathrm{M}+\mathrm{Na}]^{+}$. Anal. Calcd for $\mathrm{C}_{26} \mathrm{H}_{28} \mathrm{O}_{6}(E, Z$ mixture): C, 71.54; H, 6.47. Found: C, 71.94; H, 6.39.

Sporiolide A (1). To a suspension of $\mathrm{Pd} / \mathrm{C}(5 \% \mathrm{Pd}, 15 \mathrm{mg})$ in $\mathrm{MeOH}(8 \mathrm{~mL})$ was added a solution of $4(45 \mathrm{mg}, 0.1 \mathrm{mmol})$ in $\mathrm{MeOH}(4 \mathrm{~mL})$. The mixture was allowed to stir at room temperature for $24 \mathrm{~h}$ under $4 \mathrm{~atm}$ of $\mathrm{H}_{2}$ pressure, at the end of which of time TLC indicated that the reaction was complete. The mixture was filtered and concentrated in vacuo. The residue was subjected to column chromatography on silica gel (petroleum ether-EtOAc, 7:1) to furnish sporiolide $\mathrm{A}(\mathbf{1}, 33 \mathrm{mg}, \mathbf{9 1 \%})$ as white amorphous solid: 
$[\alpha]^{25} \mathrm{D}-15(c \quad 0.5, \mathrm{MeOH}) ;{ }^{1} \mathrm{H}$ NMR $\left(\mathrm{CDCl}_{3}\right) \delta 1.16-1.21(\mathrm{~m}$, $1 \mathrm{H}), 1.23-1.28(\mathrm{~m}, 1 \mathrm{H}), 1.30(\mathrm{~d}, 3 \mathrm{H}, J=5.8 \mathrm{~Hz}), 1.35-1.45(\mathrm{~m}$, $3 \mathrm{H}), 1.58-1.73(\mathrm{~m}, 3 \mathrm{H}), 1.84-1.87(\mathrm{~m}, 1 \mathrm{H}), 2.11-2.15(\mathrm{~m}, 1 \mathrm{H})$, $2.96(\mathrm{~d}, 1 \mathrm{H}, J=17.0 \mathrm{~Hz}), 3.50(\mathrm{dd}, 1 \mathrm{H}, J=10.6,17.2 \mathrm{~Hz}), 4.39$ $(\mathrm{t}, 1 \mathrm{H}, J=4.4 \mathrm{~Hz}), 4.92-4.95(\mathrm{~m}, 1 \mathrm{H}), 5.96(\mathrm{~d}, 1 \mathrm{H}, J=10.0$ $\mathrm{Hz}), 7.48$ (t, 2H, $J=7.4 \mathrm{~Hz}), 7.62(\mathrm{t}, 1 \mathrm{H}, J=7.1 \mathrm{~Hz}), 8.05$ (d, $2 \mathrm{H}, J=7.8 \mathrm{~Hz}) ;{ }^{13} \mathrm{C} \mathrm{NMR}\left(\mathrm{CDCl}_{3}\right) \delta 19.8,20.7,26.5,30.5,33.3$, 39.9, 68.0, 73.5, 75.6, 128.3, 128.6, 129.9, 133.8, 165.4, 168.2, 207.6; $\mathrm{ESI}(+)-\mathrm{MS}$ calcd for $\mathrm{C}_{19} \mathrm{H}_{24} \mathrm{O}_{6} 348.16$ [M], found 371.57
$[\mathrm{M}+\mathrm{Na}]^{+}$. Anal. Calcd for $\mathrm{C}_{19} \mathrm{H}_{24} \mathrm{O}_{6}: \mathrm{C}, 65.50 ; \mathrm{H}, 6.94$. Found: C, 65.21; H, 6. 88 .

Acknowledgment. This work was supported by National Basic Research Program of China (2003CB415001) and NNSF of China (Project 20372081).

Supporting Information Available: Spectra for compounds 18 , 19, 20, 9, 21, 22, 23, 24, 25, 26, 5, 4, and 1 . This material is available free of charge via the Internet at http://pubs.acs.org.

JO0615504 